\title{
Determination of sliding and twinning shear stress during microindentation of Hadfield steel single crystals
}

\author{
D. V. Lychagin ${ }^{\dagger, 1}$, A. V. Filippov², O. S. Novitskaya ${ }^{2}$, A. V. Kolubaev² \\ †dvl-tomsk@mail.ru \\ ${ }^{1}$ Tomsk State University, 36 Lenin Av., Tomsk, 634050, Russia \\ ${ }^{2}$ Institute of Strength Physics and Materials Science SB RAS, 2/4 Alademichesky Av., Tomsk, 634055, Russia
}

\begin{abstract}
There is a methodological task of conformance of the results of tests of micro- and nanohardness using indenters of different shapes. When measuring the hardness of crystallographically anisotropic materials, such as single crystals or individual grains of a polycrystal, it is necessary to take into account how the orientation of the pyramid tip affects the results obtained. Investigations were carried out for a tip with four faces (Vickers pyramid) when measuring the microhardness on single crystals of Hadfield steel. The hardness measurement was performed for the $\{110\}$ and $\{112\}$ crystallographic planes and different orientations of the pyramid faces. The nominal effective shear stresses of slip and twinning systems were calculated for the cases under consideration. The calculation results were compared with the orientation of the shear traces on the face surface near the indentation of the Vickers indenter. Shear traces are formed during deformation along the maximally loaded slip or twinning systems. Around the indentation, one can observe traces of displacement from all octahedral planes possible for a given crystallographic orientation of the surface. Deformation domains are observed near the faces of the pyramid. Shear domains are regions of the crystal in which separate shear systems operate from the possible ones. The acting shear systems depend on the geometrical arrangement of the indenter faces relative to the close-packed planes. Only in some cases can one assert the predominant role of twinning. As a rule, an analysis of shear traces does not allow one to unambiguously judge the predominance of the slip or twinning mechanism in the local region near the indenter imprint. The operating systems of sliding and twinning determine the features of deformation near the indentation and its shape change.
\end{abstract}

Keywords: single crystal, microhardness, Hadfield steel, shear bands.

УДК: 539.379 .4

\section{Определение напряжения скольжения и двойникования} при микроиндентировании монокристаллов стали Гадфильда

\author{
Лычагин Д. В. ${ }^{\dagger,}$, Филиппов А. В. ${ }^{2}$, Новицкая О. С. ${ }^{2}$, Колубаев А. В. ${ }^{2}$ \\ ${ }^{1}$ Национальный исследовательский Томский государственный университет, пр. Ленина 36, Томск, 634050, Россия \\ ${ }^{2}$ Институт физики прочности и материаловедения СО РАН, пр. Академический, 2/4, Томск, 634055, Россия
}

Методическую задачу представляет согласование результатов испытаний микро- и нанотвердости инденторами разной формы. При измерениях твердости кристаллографически анизотропных материалов, таких как монокристаллы или отдельные зерна поликристалла, требуется учесть, как влияет ориентация наконечника в форме пирамиды на полученные результаты. Исследования проводили для наконечника с четырьмя гранями (пирамидка Виккерса) при измерениях микротвердости на монокристаллах стали Гадфильда. Измерение твердости было выполнено для кристаллографических плоскостей $\{110\}$ и $\{112\}$ и разных ориентаций граней пирамидки. Был произведен расчет номинальных действующих напряжений сдвига систем скольжения и двойникования для рассматриваемых случаев. Результаты расчетов сопоставили с ориентацией следов сдвига на поверхности грани около отпечатка индентора Виккерса. Следы сдвига образуются при деформации по максимально нагруженным системам скольжения или двойникования. Вокруг отпечатка можно наблюдать следы сдвига от всех октаэдрических плоскостей возможных для данной кристаллографической ориентации поверхности. Деформационные домены наблюдаются около граней пирамидки. Домены сдвига - это области кристалла, в которых действуют отдельные системы сдвига из возможных. Действующие системы сдвига зависят от геометрического расположения граней индентора 
относительно плотноупакованных плоскостей. Только в отдельных случаях можно утверждать о преимущественной роли двойникования. Как правило, анализ следов сдвига не позволяет однозначно судить о преобладании механизма скольжения или двойникования в локальной области вблизи отпечатка индентора. Действующие системы скольжения и двойникования определяют особенности деформации вблизи отпечатка и его формоизменение.

Ключевые слова: монокристалл, микротвердость, сталь Гадфильда, следы сдвига.

\section{1. Введение}

Измерение твердости в широком диапазоне нагрузок и глубин вдавливания индентора является широко используемым методом механических испытаний. Результаты испытаний дают информацию об интегральных механических параметрах материала, неоднородностях свойств, связанных с микроструктурой, и информацию о тонких механизмах деформации. Измерения на микроструктурном уровне дополняются результатами чувствительности свойств на уровне нанодиапазона. В последнем случае получают данные о начальной и упругой деформации. Исследованиям упругопластического внедрения наконечника в монокристаллы посвящены работы Bishop R. F. et al. [1], Marsh D. M. [2], Johnson K.L. [3], Armstrong R. W. et al. [4], Mao Liu [5] и других исследователей. Данные испытания позволяют делать выводы о влиянии кристаллографической ориентации на твердость.

Для анализа структурных изменений при индентировании важным является понимание распределения напряжений перед индентором. Большая часть результатов получена путем моделирования напряженного состояния методом конечных элементов [5-9]. В зоне контакта индентора с материалом возникает неоднородное поле напряжений, которое зависит от формы индентора. Вблизи контактирующих поверхностей основные напряжения сжимающие, однако, в близлежащей зоне может формироваться небольшой участок растягивающих напряжений. По мере удаления от поверхности индентора напряжения экспоненциально затухают. Основная часть результатов получена для конического индентора с разным радиусом закругления. Наряду c развитием методики индентирования структура разориентации в зоне внедрения индентора исследуется методами EBSD $[5,9,10]$ и TEM [5,11]. С помощью измерения микротвердости на монокристаллах исследуют влияние кристаллографической ориентации на механические свойства. Исследования твердости проведены на монокристаллах алюминия [5], меди $[5,7,12]$, золота [13], жаропрочного сплава [8], аустенитной стали $[10,11]$ и других материалах $[14,15]$. При измерении микротвердости поликристаллов есть возможность учитывать влияние кристаллографической ориентации зерен при контроле их ориентации методом EBSD [16], а также совмещающие методы индентирования и акустической эмиссии [17]. Исследования на ГЦК монокристаллах показали, что наблюдается зависимость между величиной нано- и микро- твердости, упругости и кристаллографической ориентацией плоскости нагружения. Характер микроструктуры возле индентора, исследованный методом EBSD и TEM, хорошо соот- ветствует, рассчитанному методом конечных элементов, полю напряжений в этой области. Распределение напряжений вблизи конического индентора согласуется c анизотропией сдвига данной ориентации монокристаллов. В рассматриваемых выше материалах деформация развивается скольжением. Однако имеется круг материалов, в которых наряду со скольжением развивается двойникование. К таким сталям относится сталь Гадфильда. Эта сталь является представителем материалов, где реализуется ориентационная зависимость этих механизмов. Это должно находить свое отражение при испытаниях твердости. Целью настоящей работы является изучение ориентационной зависимости деформационных процессов при микроиндентировании монокристаллов стали Гадфильда.

\section{2. Материал и методика}

В качестве материала исследования была выбрана сталь Гадфильда 110Г13 следующего химического состава: $\mathrm{C}-1.1 \%, \mathrm{Mn}-12.5 \%, \mathrm{Si}-0.4 \%, \mathrm{Ni}-0.15 \%, \mathrm{Cr}-0.29 \%$, $\mathrm{V}-0.035 \%$, Сo - 0.04\%, Ti - 0.007\%, Fe -остальное. Монокристаллы выращивали по методу Бриджмена в атмосфере гелия и затем гомогенизировали в инертном газе 24 часа при 1373 К. Испытания на микротвердость проводили на пластинах толщиной 5 мм и размером поверхности $20 \times 20$ мм. Поверхность подвергали шлифовке и электролитической полировке. Ориентацию контролировали методом дифракции обратно отраженных электронов на сканирующем электронном микроскопе Tescan Mira 3. Исследуемая поверхность граней имела ориентации (001) и (112). Для каждой грани делали по два укола с поворотом индентора на $45^{\circ}$. Испытания проводили на микротвердомере CSM Instrument индентором Виккерса с максимальной нагрузкой 10 Н. Анализ деформационного рельефа выполнен с помощью лазерного сканирующего микроскопа Olympus OLS LEXT 4100.

\section{3. Расчет напряжений скольжения и двойникования}

При внедрении пирамидального индентора основное влияние на развитие сдвига оказывают его грани, поэтому при расчетах можно пренебречь неоднородностью напряжений за счет ребер индентора, как первоначального концентратора напряжений. Для определения наиболее нагруженных систем скольжения и двойникования для каждого случая ориентации граней пирамидки при известной кристаллографической ориентации поверхности индентирования были проведены расчеты по представленной далее методике. 
Сила нагрузки $F_{n}$ прикладывается ортогонально плоскости монокристалла. Поскольку пирамидка имеет четыре одинаковые плоских грани, то можно принять, что действие силы нормальной нагрузки распределяется равномерно между этими площадками. При значении нормальной силы $F_{n}=10 \mathrm{H}$ на каждую площадку действует сила $2.5 \mathrm{H}$. Площадь каждой грани индентора рассчитывали исходя из значения стороны отпечатка $b$, связанного тригонометрически с высотой грани $h$. Напряжение, действующее со стороны каждой из граней индентора, рассчитывали как отношение $1 / 4 F_{n}$ к площади грани пирамидки.

Известная геометрия пирамидки и кристаллографическая ориентация поверхности нагрузки позволяет вычислить кристаллографические направления, вдоль которых действуют силы от каждой площадки при определенной ориентации поверхностей пирамидки. Для поверхности (001) при ориентации пирамидки, дающей отпечаток № 1, это кристаллографические направления:

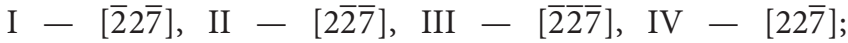
отпечаток № 2: I - $[\overline{0} 2 \overline{5}]$, II $-[\overline{0} 2 \overline{5}]$, III $-[\overline{0} 2 \overline{5}]$, IV -

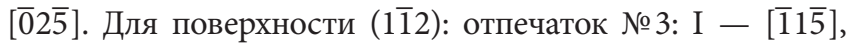

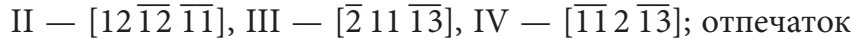
№ 4: I $-[\overline{4} 1 \overline{12}]$, II $-[\overline{5} 7 \overline{6}]$, III $-[\overline{1} 10 \overline{16}]$, IV $-[\overline{3} 1 \overline{3}]$.

С использованием данных по действию силы от каждой грани были определены номинальные действующие напряжения скольжения и двойникования для возможных систем скольжения и векторов Бюргерса частичной дислокации ГЦК кристаллов для двух ориентаций отпечатков № 1 и № 2 на поверхности (001) (Табл. S1, дополнительный материал). Аналогично проведен рас-

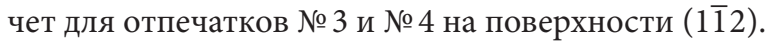

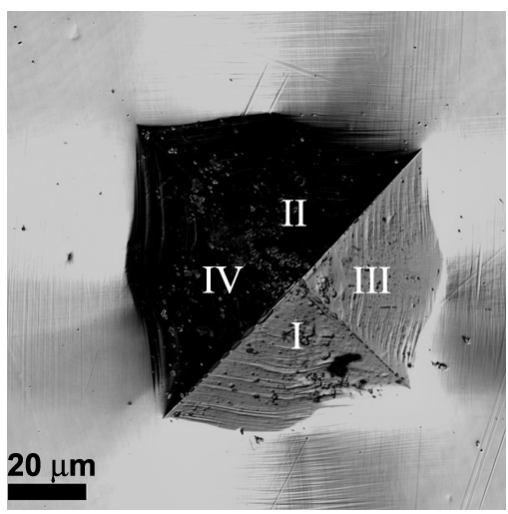

a

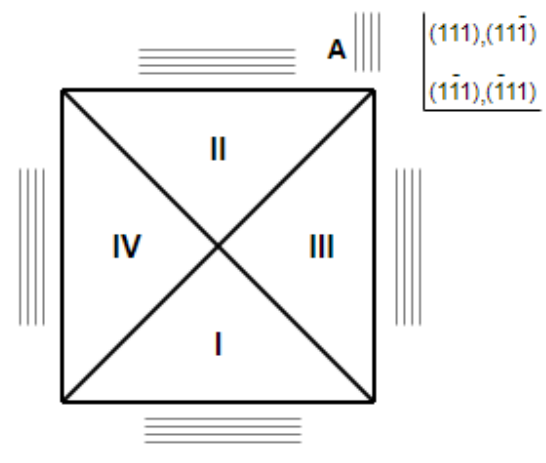

c

\section{4. Экспериментальные результаты и обсуждения}

\section{1. Грань (001)}

На Рис. 1 приведены изображения отпечатков и деформационного рельефа, полученные после индентирования грани (001) с разной ориентацией граней пирамидки относительно следов выхода плотноупакованных плоскостей на поверхность грани.

Рядом представлены схемы расположения следов относительно поверхности отпечатка и схема выходов плотноупакованных плоскостей. Последнее позволяет определить индексы плотноупакованной плоскости, формирующей след сдвига, и сопоставить картину сдвига с результатами расчета.

На основании анализа следов скольжения можно сделать вывод, что в области вокруг отпечатка деформация осуществляется по всем четырем равнонагруженным октаэдрическим плоскостям. Однако, в локальной области вблизи отпечатка № 1 наблюдается только одна система следов, около отпечатка №2 - две (Рис. 1). При этом, геометрия сдвига такова, что один след может быть сформирован сдвигом по двум октаэдрическим плоскостям.

Для того чтобы выяснить какие плоскости и системы сдвига являются наиболее приоритетными, а также определить возможные механизмы деформации, нужно воспользоваться результатами расчета напряжений для силы нормальной грани пирамидки (Табл. S1, дополнительный материал). Из данных расчета следует, что при об-

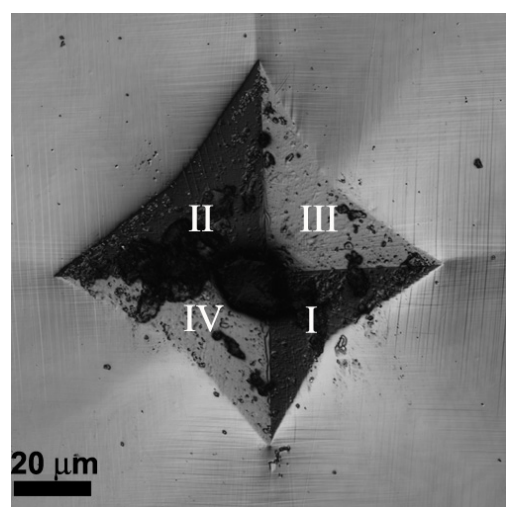

$\mathrm{b}$

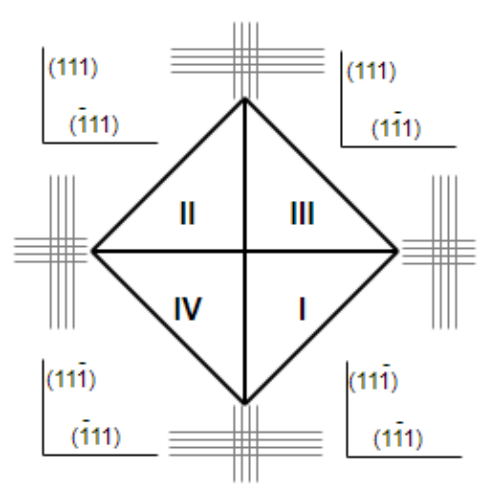

d

Рис. 1. Деформационный рельеф (a, c) и схема следов скольжения (b, d) для отпечатка № 1 (a, b), отпечатка № 2 (c, d). Fig. 1. Strain relief (a, c) and slip pattern (b, d) for print No. 1 (a, b), print No. 2 (c, d). 
разовании отпечатка № 1 наблюдаются только такие следы деформации, для которых максимально напряжение двойникования по одной системе. В отдельных областях возможно развитие деформации скольжением. Такой случай, по-видимому, реализуется на стыке граней II и III. Кроме этого, известно [18], что в монокристаллах стали Гадфильда при сжатии в [001] деформация развивается двойникованием, а при растяжении - скольжением. В этом случае фактор Шмида максимален для восьми равнонагруженных систем скольжения. Ориентация отпечатка совпадает с ориентацией октаэдра скольжения в направлении [001], а грани располагаются вдоль направлений $\{110\}$. В этом случае сдвиг от каждой грани должен давать одну систему следов скольжения, что и наблюдается экспериментально. Системы октаэдрических плоскостей параллельные грани пирамидки способствуют расширению стороны отпечатка.

Отпечаток № 2. Поворот индентора на $45^{\circ}$ делает возможным активизацию двух систем следов от каждой грани пирамидки. При выбранном расположении плоскостей сдвига $\{111\}$ относительно отпечатка основные следы скольжения формируются у острия отпечатка. Теоретический анализ максимальных напряжений сдвига показывает, что при переходе через острие отпечатка плоскости октаэдра, располагающиеся перпендикулярно острию изменяются, а параллельные остаются неизменными (Табл. S1, дополнительный материал). Это отражено на схеме на Рис. 1d. Меняющаяся октаэдрическая плоскость соответствует смежной плотноупакованной плоскости в октаэдре скольжения. В результате для каждого участка грани отпечатка выбирается наиболее оптимальная пара равнонагруженных плоскостей октаэдрического сдвига. Совокупное действие данных октаэдрических плоскостей обеспечивает преимущественную деформацию в направлении каждого острия отпечатка. При этом из картины деформационного рельефа разделить механизмы на скольжение и двойникование не представляется возможным.

Результаты анализа хорошо согласуются экспериментально наблюдаемой картиной локализации следов сдвига на грани (001) (Рис. 1). Форма отпечатков № 1 и № 2 обусловлена геометрией расположения активных октаэдрических плоскостей сдвига относительно отпечатка.

\section{2. Грань (11̄2)}

При ориентации отпечатков № 3 и № 4 на грани (1푸) относительно плоскостей $\{111\}$ кристаллографические направления нормалей к граням индентора будут

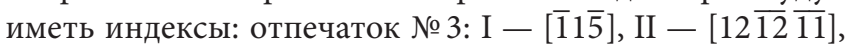
III $-[\overline{2} 11 \overline{13}]$, IV $-[\overline{11} 2 \overline{13}]$; отпечаток № $4:$ I $-[\overline{4} 1 \overline{12}]$, $\mathrm{II}-[\overline{5} 7 \overline{6}], \mathrm{III}-[\overline{1} 10 \overline{16}], \mathrm{IV}-[\overline{3} 1 \overline{3}]$.Эти направления соответствуют направлению силы, действующей от каждой грани отпечатка. Рассчет напряжений скольжения и двойникования для этих сил выполнен аналогично грани (001).

Отпечаток № 3. Расчеты показали, что наибольшее число действующих систем сдвига с максимальным значением напряжений скольжения и двойникования будет действовать вблизи грани I. При этом, практически в каждой плоскости возможна деформация как скольжением, так и двойникованием. В картине деформационного рельефа вблизи грани I это находит свое отражение в формировании двух систем следов (Рис. 2).

На представленной рядом схеме показано расположение следов скольжения и указаны индексы плотноупакованных плоскостей, формирующих эти следы. Это следы от плоскостей (111) и (11 $\overline{1})$. У остальных граней преобладают следы от деформации по плоскостям (111). Около граней III и IV наблюдаются небольшие участки со второй системой. Однако, более детальное рассмотрение требует анализа первичного и вторичного формирования следов. Образование следов от деформации на втором этапе может происходить в других условиях: может изменяться ориентация, локальные напряжения, механизм деформации.

Отпечаток № 4. На Рис. 3 показан деформационный рельеф на грани (1다) с отпечатком после поворота пирамидки и схема для этого случая.

Формируется большее число деформационных доменов с системами следов от трех октаэдрических плоскостей. Это согласуется с экспериментальными резуль-

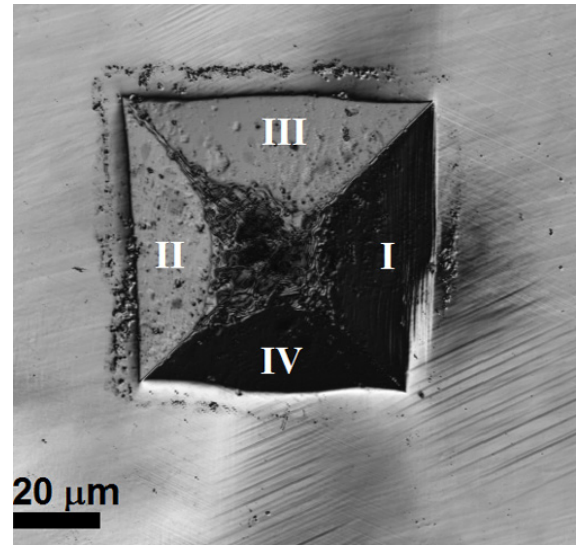

a

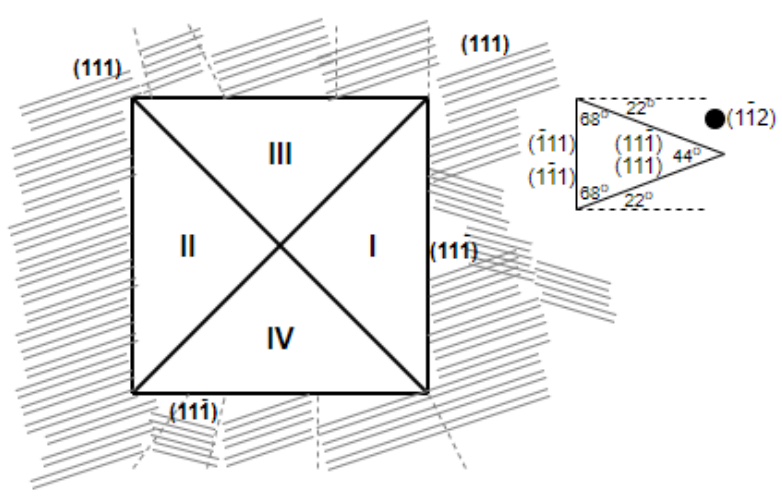

b

Рис. 2. Деформационный рельеф (а) и схема следов скольжения (b) для отпечатка № 3.

Fig. 2. Strain relief (a) and slip pattern (b) for print No. 3. 


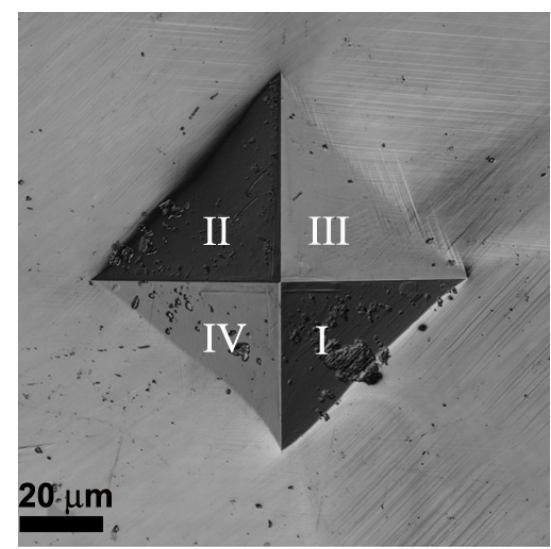

a

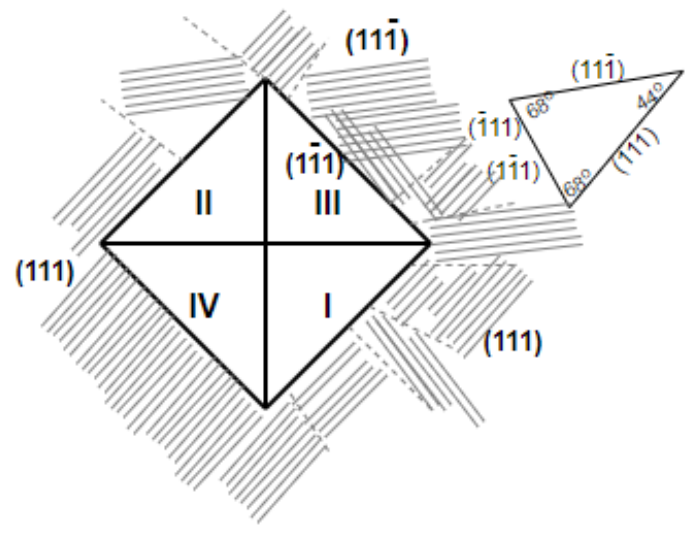

b

Рис. 3. Деформационный рельеф (а) и схема следов скольжения (b) для отпечатка № 4.

Fig. 3. Strain relief (a) and slip pattern (b) for print No. 4 .

татами. В большинстве из рассматриваемых плоскостей деформация может идти как по механизму дислокационного скольжения, так и по механизму механического двойникования.

Значение твердости при индентировании обусловлено сопротивлением деформации при внедрении. Это сопротивление определяется механизмами упрочнения. Вклад за счет субструктурного упрочнения связан с взаимодействием дислокаций одной системы скольжения с дислокациями другой, двойниками, а также взаимодействием двойников разных систем. Размер области пересечения следов от разных систем скольжения определяет степень влияния такого вклада. По-видимому, с этим связано то, что для отпечатков на грани (1/12) (отпечатки № 3 и № 4, диагональ отпечатка 65-66 мкм) твердость выше, чем для грани (001) (отпечатки № 1 и № 2, диагональ отпечатка 71- 72 мкм).

\section{5. Заключение}

Таким образом, на основании проведенных расчетов выполнен анализ действующих напряжений и возможных механизмов деформации для монокристаллов стали Гадфильда с различной кристаллографической ориентацией индентора при микроиндентировании.

Проанализирована активность возможных систем сдвига для деформации по механизму дислокационного скольжения и двойникования, которая зависит от кристаллографической ориентации граней пирамидки Виккерса. Наблюдается полное соответствие между теоретически определенными системами скольжения и реальной картиной деформационного рельефа (локализацией и ориентацией следов скольжения относительно граней отпечатка).

Установлено, что для каждого из рассматриваемых случаев ориентации индентора возможна реализация как скольжения, так и двойникования. Исходя из анализа действующих напряжений, можно предположить, что для отпечатка №1 (грань (001)) самая высокая вероятность двойникования.
Деформация около внедряемой пирамидки развивается фрагментировано с образованием деформационных доменов, локальных областей, в которых деформация развивается преимущественно по одной системе скольжения или двойникования. Расположение деформационных доменов зависит от поля напряжения вблизи индентора, определяемого его формой (локальным тензором напряжений) и ориентацией систем октаэдрического сдвига и двойникования относительно этого поля.

Дополнительныйматериал/Supplementarymaterial. Электронная версия статьи содержит дополнительный материал (Табл. S1), доступный безвозмездно на сайте журнала (lettersonmaterials.com). / The online version of this paper contains supplementary material (Table S1) available free of charge at the journal's Web site (lettersonmaterials.com).

Благодарности/Acknowledgements. Авторы выражают благодарность профессору Ю.И. Чумлякову за предоставленные для исследований монокристалль. Работа выполнена в рамках государственного задания ИФПМ СО РАН, проект III.23.2.4. Исследования проводились с использованием оборудования центра коллективного пользования "Аналитический центр геохимии природных систем» НИ ТГУ. / Authors of the article express their gratitude to Professor Yu.I. Chumlyakov for single crystals of steel. The work was performed according to the Government research assignment for ISPMS SB RAS, project No. III.23.2.4. Research were performed using the equipment of Tomsk center of collective use "Analytical Center for Geochemistry of Natural Systems» NR TSU.

\section{Литература/References}

1. R. F. Bishop, R. Hill, N. F. Mott. Proc. Phys. Soc. 57, 147 (1945). Crossref

2. D. M. Marsh. Proc. Roy. Soc. A. 279, 420 (1964).

3. K. L. Johnson. Contact mechanics. Cambridge University Press (1985) 452 p. $\underline{\text { Crossref }}$

4. R. W. Armstrong, W.L. Elban, S. M. Walley. Int. J. Mod. 
Phys. B. 27 (8), 1330004 (2013). Crossref

5. M.Liu. Crystal plasticity and experimental studies of nanoindentation of aluminium and copper: Ph. D. Thesis. University of Wollongong, Australia (2014).

6. J. Alcala, O. Casals, J. Ocenasek. J. Mech. Phys. Solids. 56, 3277 (2008). Crossref

7. Y. Liu, B. Wang, M. Yoshino, S. Roy, H. Lu, R. Komanduri. J. Mech. Phys. Solids. 53, 2718 (2005). rossref

8. B. Eidel. Acta Mater. 59, 1761 (2011). Crossref

9. N. Zaafarani, D. Raabe, R. N. Singh, F. Roters, S. Zaefferer. Acta Mater. 54, 1863 (2006). Crossref

10. S. Chen, Y. Miyahara, A. Nomoto. Phil. Mag. Lett. 98 (11), 473 (2018). Crossref

11. T. Chen, L. Tan, Z. Lu, H. Xu. Acta Mater. 138, 83 (2017). Crossref

12. M. Rester, C. Motz, R. Pippan. Scripta Mater. 59, 742 (2008). Crossref
13. M. M. McCann. Nanoindentation of Gold Single Crystals: Ph.D Thesis. Virginia Polytechnic Institute and State University, Blacksburg (2004).

14. A.I. Tyurin, S.D. Victorov, A.N. Kochanov, A. V. Shuklinov, T.S. Pirozhkova. AIP Conf. Proc. 1783, 020227 (2016). Crossref

15. N. L. Sizova. Crystallography Reports. 61 (3), 425 (2011). (in Russian) [Н.Л. Сизова. Кристаллография. 61 (3), 425 (2011).] Crossref

16. B. Lv, M. Zhang, F.C. Zhang, C.L. Zheng, X. Y. Feng, L.H. Qian, X.B. Qin. Int.J. Fatigue. 44, 273 (2012). Crossref

17. D. L. Merson, D. E. Mesheryakov, A. Vinogradov. Progress in acoustic emission XIV. The Japanese Society for NDI (2008) pp. 115-120.

18. I. Karaman, H. Sehitoglu, K. Gall, Y.I. Chumlyakov, H. J. Maier. Acta Mater. 48, 1345 (2000). $\underline{\text { Crossref }}$ 